\title{
Immunophenotypic diversity of endometrial adenocarcinomas: implications for differential diagnosis
}

\author{
Michelle Reid-Nicholson ${ }^{1, *, * *}$, Pratibha Iyengar ${ }^{1, * *, \dagger}$, Amanda J Hummer ${ }^{2}$, Irina Linkov ${ }^{3}$, \\ Marina Asher ${ }^{1}$ and Robert A Soslow ${ }^{1}$ \\ ${ }^{1}$ Department of Pathology, Memorial Sloan-Kettering Cancer Center, New York, NY, USA; ${ }^{2}$ Department \\ of Epidemiology and Biostatistics, Memorial Sloan-Kettering Cancer Center, New York, NY, USA and \\ ${ }^{3}$ Immunohistochemistry Core Laboratory, Memorial Sloan-Kettering Cancer Center, New York, NY, USA
}

\begin{abstract}
Many endometrial adenocarcinomas, particularly those of endometrioid type, express estrogen receptors (ERs), progesterone receptors (PRs), and vimentin. This typical immunophenotype is frequently considered a standard against which others are compared when immunohistochemistry is used for differential diagnosis. We tested large numbers of endometrial cancers, enriched for high-grade tumors, to determine whether this reported immunophenotype was valid and whether expression differences between types of endometrial carcinoma could be exploited for diagnostic purposes. Immunohistochemical stains were performed on the following types of endometrial cancers using established methodology: International Federation of Gynecology and Obstetrics (FIGO) grades 1 and 2 endometrioid-42; FIGO grade 3 endometrioid-40; serous-24; clear cell-11; carcinosarcoma-9. In total, $92 \%$ of serous carcinomas expressed p16 strongly compared to weak-tomoderate expression of p16 in 7-67\% of other tumors (FIGO grades 1 and 2 carcinoma and carcinosarcoma, respectively). A total of $84 \%$ of FIGO grades 1 and 2 carcinomas expressed ER compared to $9-54 \%$ of other tumors (clear cell and serous carcinomas respectively); 83\% of FIGO grades 1 and 2 expressed PR compared to $\mathbf{1 1 - 5 4 \%}$ of other carcinomas (carcinosarcoma and serous carcinoma, respectively). Most carcinomas were negative for monoclonal carcinoembryonic antigen (mCEA), and those that were positive showed mostly only focal membrane expression. Vimentin was expressed in nearly every tumor. Most tumors were diffusely vimentin positive, but a large range of expression patterns, from focal to diffuse and from weak to strong, was noted. Only $70 \%$ of FIGO grades 1 and 2 endometrioid carcinomas and $26 \%$ of grade 3 endometrioid carcinomas possessed the reportedly characteristic endometrial cancer immunophenotype p16 (-), ER (+), PR (+), mCEA $(-)$, and vimentin $(+)$. Endometrial cancers demonstrate substantial immunophenotypic diversity that remained apparent even within groups of similar histologic subtype and grade. ER, PR, and p16 expression was more illustrative of tumor type and degree of differentiation than they were of endometrial origin. In contrast, the vimentin-positive/CEA-negative phenotype remained the most constant among all endometrial cancers.
\end{abstract} Modern Pathology (2006) 19, 1091-1100. doi:10.1038/modpathol.3800620; published online 28 April 2006

Keywords: endometrial cancer; p16; serous carcinoma; immunohistochemistry

It remains a diagnostic challenge to discriminate between some endometrial and endocervical adenocarcinomas, especially in curettage material.

Correspondence: Dr RA Soslow, MD, Department of Pathology, Memorial Sloan-Kettering Cancer Center, 1275 York Avenue, C524, New York, NY 10021, USA.

E-mail: soslowr@mskcc.org

${ }^{*}$ Current address: Department of Pathology, Medical College of Georgia, Augasta, GA, USA.

**Both authors contributed equally to this work and are considered co-first authors.

Current address: Department of Laboratory Medicine, Credit Valley Hospital, Mississauga, ON, Canada.

Received 9 March 2006; revised 23 March 2006; accepted 31 March 2006; published online 28 April 2006
Morphologically similar carcinomas, such as endocervical adenocarcinomas, have been reported to express p16 $16^{1-3}$ and carcinoembryonic antigen (CEA), ${ }^{4-6}$ whereas endometrial adenocarcinomas frequently show estrogen receptor (ER), ${ }^{7,8}$ progesterone receptor (PR), ${ }^{7,8}$ and vimentin expression. ${ }^{4,7,9}$ In practice, many pathologists have used immunohistochemical panels composed of markers against these antigens for determining site of origin. In most cases, this approach is not problematic because endometrioid adenocarcinomas usually derive from the uterine corpus. However, there is substantial morphologic heterogeneity within each tumor family related to types and degree of differentiation, which usually means that there exists considerable 
immunophenotypic variation within each tumor family. Another problem with using this approach is that there are several different tumor types other than endometrioid adenocarcinoma that arise in the endometrium, potentially confusing site assessment. We therefore hypothesized that an immunohistochemical profile is likely to be informative about tumor type and degree of differentiation, but not necessarily about site of origin. We tested large numbers of endometrial cancers, enriched for highgrade tumors, to determine whether the ER/PR/ vimentin-positive, p16/CEA-negative immunophenotype was valid and whether expression differences between types of endometrial carcinoma could be exploited for diagnostic purposes.

\section{Materials and methods}

Immunohistochemical stains were performed on the following types of endometrial cancers using established methodology: International Federation of Gynecology and Obstetrics (FIGO) grades 1 and 2 endometrioid carcinomas, 42; FIGO grade 3 endometrioid carcinomas, 40; serous carcinomas, 24; clear cell carcinomas, 11; and carcinosarcoma, 9.

\section{Study Population}

Patients undergoing surgery for gynecologic malignancies at Memorial Sloan-Kettering Cancer Center (MSKCC) had their tumor specimens banked under an Institutional Review Board (IRB)-approved tissue acquisition protocol after giving their informed consent. Tumor microarrays were constructed using micro-core hysterectomy tumor specimens from patients whose tissues were selected to provide a broad representation of types, grades, and stages of endometrial cancers. Informative immunohistochemistry data from the microarray were available for 97 patients, including 42 with FIGO grades 1 and 2 endometrioid carcinomas, 36 with FIGO grade 3 endometrioid carcinoma, four with serous carcinoma, six with clear cell carcinoma, and nine with carcinosarcoma. In an effort to expand the numbers of tumor types that were poorly represented in the microarray, we randomly selected from the surgical pathology files at MSKCC additional recent cases of endometrial serous carcinoma $(n=20)$, clear cell carcinoma $(n=5)$, and FIGO grade 3 endometrioid adenocarcinoma $(n=4)$.

\section{Pathology Review}

All tumor slides were reviewed by at least two reference pathologists. We confirmed endometrial origin, histologic subtype and grade, where applicable, of every tumor studied with immunohistochemistry.

\section{Tumor Microarrays}

Core needle biopsies of pre-existing paraffin-embedded tissue were obtained and then re-embedded in an arrayed master block using techniques originally developed by Kononen et $a l^{10}$ and then modified by Hedvat et $a l^{11}$ We used the Beecher Instruments (Sun Prairie, WI, USA) arraying device to produce sample circular spots that were $0.6 \mathrm{~mm}$ in diameter. Three core needle specimens were obtained from each tumor and companion tissue specimen.

\section{Immunohistochemistry}

Immunohistochemistry was performed according to standard protocols (Table 1). We used an immunohistochemical scoring system that took into account distribution and intensity of immunoreactivity. $1+$ results were characterized by focal staining of weak intensity. $2+$ results showed either diffuse weak staining or focal/patchy moderately intense staining. $3+$ cases demonstrated either diffuse moderately intense staining or focal/patchy intense staining. $4+$ cases were diffusely and strongly immunoreactive. Only the epithelial component of carcinosarcomas was scored. Scores of $1+$ and $2+$ are described in the manuscript as 'weak-to-moderate' and scores of $3+$ and $4+$ are described as 'strong.'

Table 1 Immunohistochemistry material and methods

\begin{tabular}{|c|c|c|c|c|c|c|}
\hline & Clone & Vendor & Location & Antigen retrieval ${ }^{\mathrm{a}}$ & Dilution & Pattern \\
\hline p16 & 16P04 & Neo Markers/Lab Vision & Fremont, CA & Citrate, pH 6.00 & $1: 400$ & $\mathrm{C}, \mathrm{N}$ \\
\hline ER & ERID5 & Beckman Coulter & Miami, FL & Citrate, pH 6.00 & $1: 100$ & $\mathrm{~N}$ \\
\hline PR & 10A9 & Beckman Coulter & Miami, FL & - & $1: 200$ & $\mathrm{~N}$ \\
\hline mCEA & A5B7 & DAKO & Carpinteria, CA & Citrate, pH 6.00 & $1: 500$ & $\mathrm{C}, \mathrm{M}$ \\
\hline Vimentin & V9 & DAKO & Carpinteria, CA & Citrate, pH 6.00 & $1: 4000$ & $\mathrm{C}$ \\
\hline
\end{tabular}

ER, estrogen receptor; PR, progesterone receptor; mCEA, monoclonal carcinoembryonic antigen; C, cytoplamsic; N, nuclear; M, membrane.

${ }^{\mathrm{a}}$ Heat-induced antigen retrieval using a microwave. 


\section{Statistical Evaluation}

Immunohistochemical results were considered negative if the score was no higher than 0 . Stains were considered positive if the score was 1 or higher. The chi-square test was used to determine a significant difference in histologic subtype and marker expression for p16, ER, PR, CEA, and vimentin.

\section{Results}

Detailed immunohistochemical results are presented in Table 2. Summaries of these data follow.

p16 (Figures 1 and 2): p16 was largely negative in FIGO grades 1 and 2 endometrioid adenocarcinomas, while $25 \%$ of FIGO grade 3 tumors were positive. In all, $92 \%$ of serous carcinomas were p16positive, and $45 \%$ of clear cell carcinomas expressed p16. A total of $67 \%$ of carcinosarcomas were positive. Tumors showing the strongest immunoreactivity were serous carcinomas and the epithelial component of carcinosarcomas. With the exception of approximately one-third of clear cell carcinomas, which were strongly labeled, all of the remaining positive tumors were weakly or moderately immunoreactive. Tumor cells demonstrating immunoreactivity showed cytoplasmic and nuclear decoration.

ER and PR (Figures 3-5): In all, 84\% of FIGO grades 1 and 2 endometrioid carcinomas expressed ER compared to $50 \%$ of FIGO grade 3 carcinomas, $54 \%$ of serous carcinomas, and $9 \%$ of clear cell carcinomas. A total of of FIGO grades 1 and 2 endometrioid carcinomas expressed PR compared to $42 \%$ of FIGO grade 3 carcinomas, $54 \%$ of serous carcinomas, $45 \%$ of clear cell carcinomas, and $11 \%$ of carcinosarcomas. Tumors showing the strongest immunoreactivity for ER and PR were endometrioid adenocarcinomas of all FIGO grades. Occasional clear cell carcinomas and serous carcinomas showed strong PR staining. When they showed any expression at all, ER expression was weak or at most moderate in clear cell carcinoma, serous carcinoma, and carcinosarcoma.

Table 2 Immunohistochemical stains by histologic subtype

\begin{tabular}{|c|c|c|c|c|c|}
\hline & $p 16(+)$ & $E R(+)$ & $P R(+)$ & $m C E A(+)$ & Vimentin (+) \\
\hline FIGO grades 1 and 2 & $7 \%(3 / 42)$ & $84 \%(31 / 37)$ & $83 \%(35 / 42)$ & $7 \%(3 / 42)$ & $90 \%(38 / 42)$ \\
\hline FIGO grade 3 & $25 \%(10 / 40)$ & $50 \%(20 / 40)$ & $42 \%(16 / 38)$ & $2.5 \%(1 / 40)$ & $81 \%(30 / 37)$ \\
\hline Serous & $92 \%(22 / 24)$ & $54 \%(13 / 24)$ & $54 \%(13 / 24)$ & $13 \%(3 / 24)$ & $83 \%(19 / 23)$ \\
\hline Clear cell & $45 \%(5 / 11)$ & $9 \%(1 / 11)$ & $45 \%(5 / 11)$ & $18 \%(2 / 11)$ & $91 \%(10 / 11)$ \\
\hline Carcinosarcoma & $67 \%(6 / 9)$ & $22 \%(2 / 9)$ & $11 \%(1 / 9)$ & $0 \%(0 / 9)$ & $100 \%(9 / 9)$ \\
\hline$P$-value* & $<0.001$ & $<0.001$ & $<0.001$ & 0.28 & 0.49 \\
\hline
\end{tabular}

ER, estrogen receptor; PR, progesterone receptor; mCEA, monoclonal carcinoembryonic antigen; FIGO, International Federation of Gynecology and Obstetrics.

${ }^{*}$ Significant $P$-values indicate differences between histologic subtypes with respect to immunophenotype.
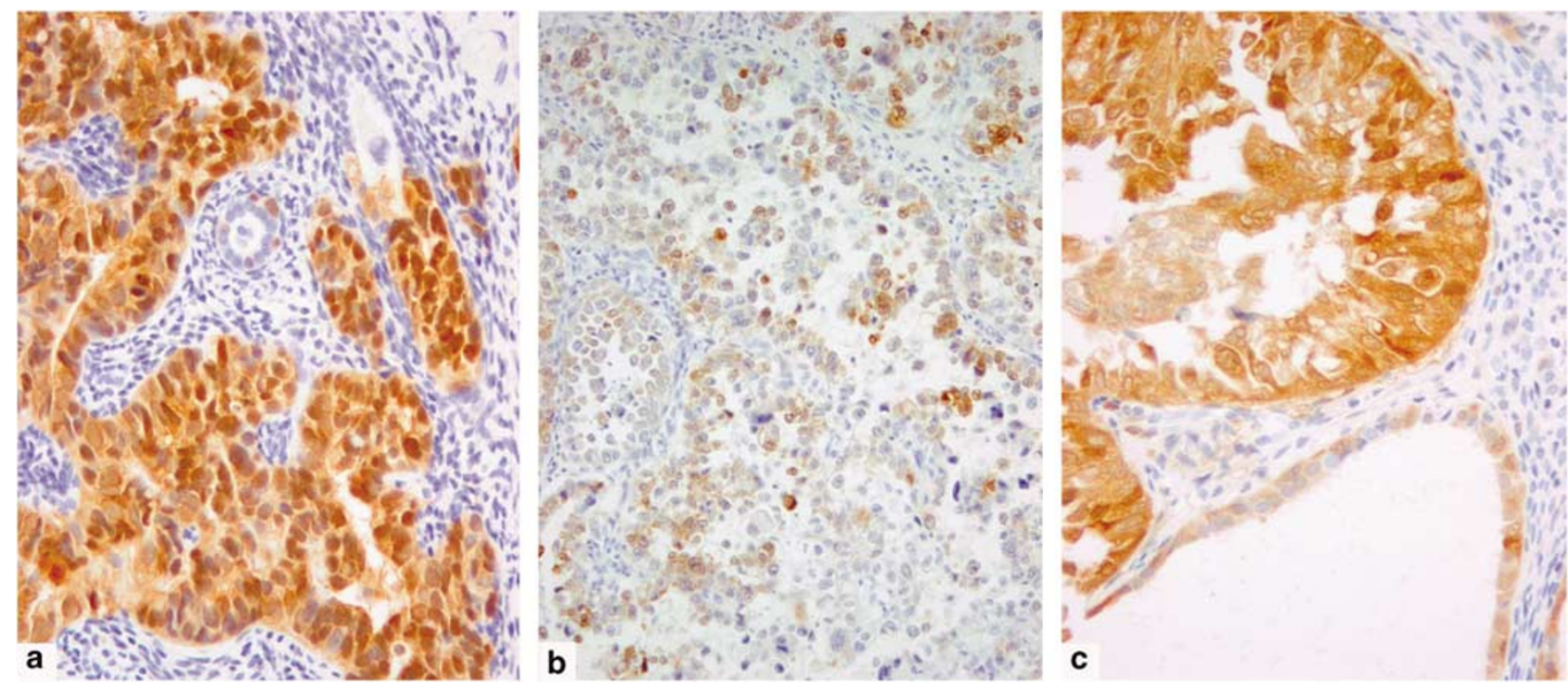

Figure 1 p16 expression in endometrial cancer. (a) Diffuse, strong, nuclear, and cytoplasmic labeling of serous carcinoma. Note p16negative atrophic endometrium (upper right), partly colonized by serous carcinoma. (b) A clear cell carcinoma with patchy p16 expression. (c) Another serous carcinoma (upper left) juxtaposed with a gland lined by tubal metaplastic cells that focally express p16. 


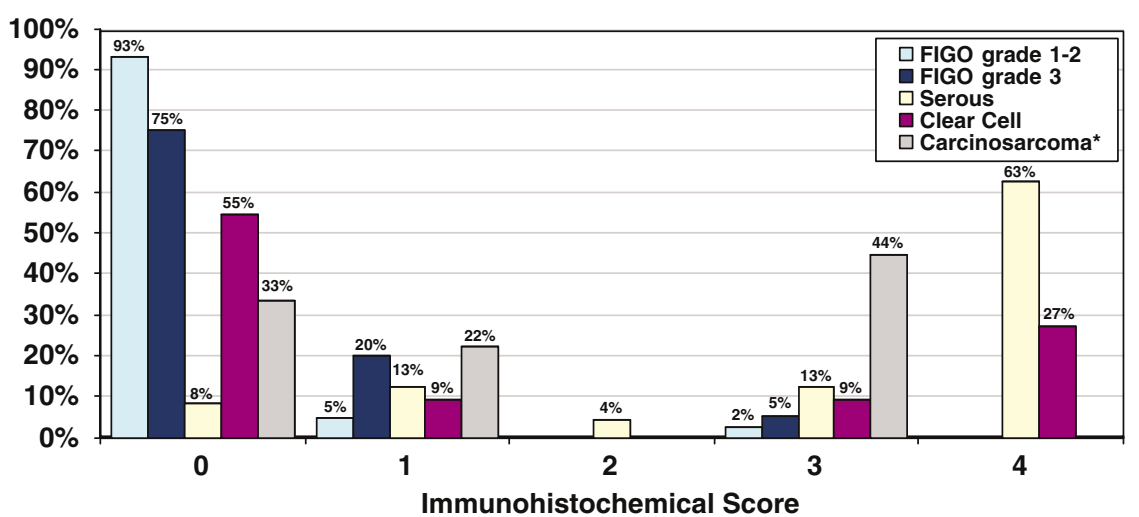

Figure 2 p16 expression in endometrial cancer. FIGO, International Federation of Gynecology and Obstetrics. *Epithelial components only.
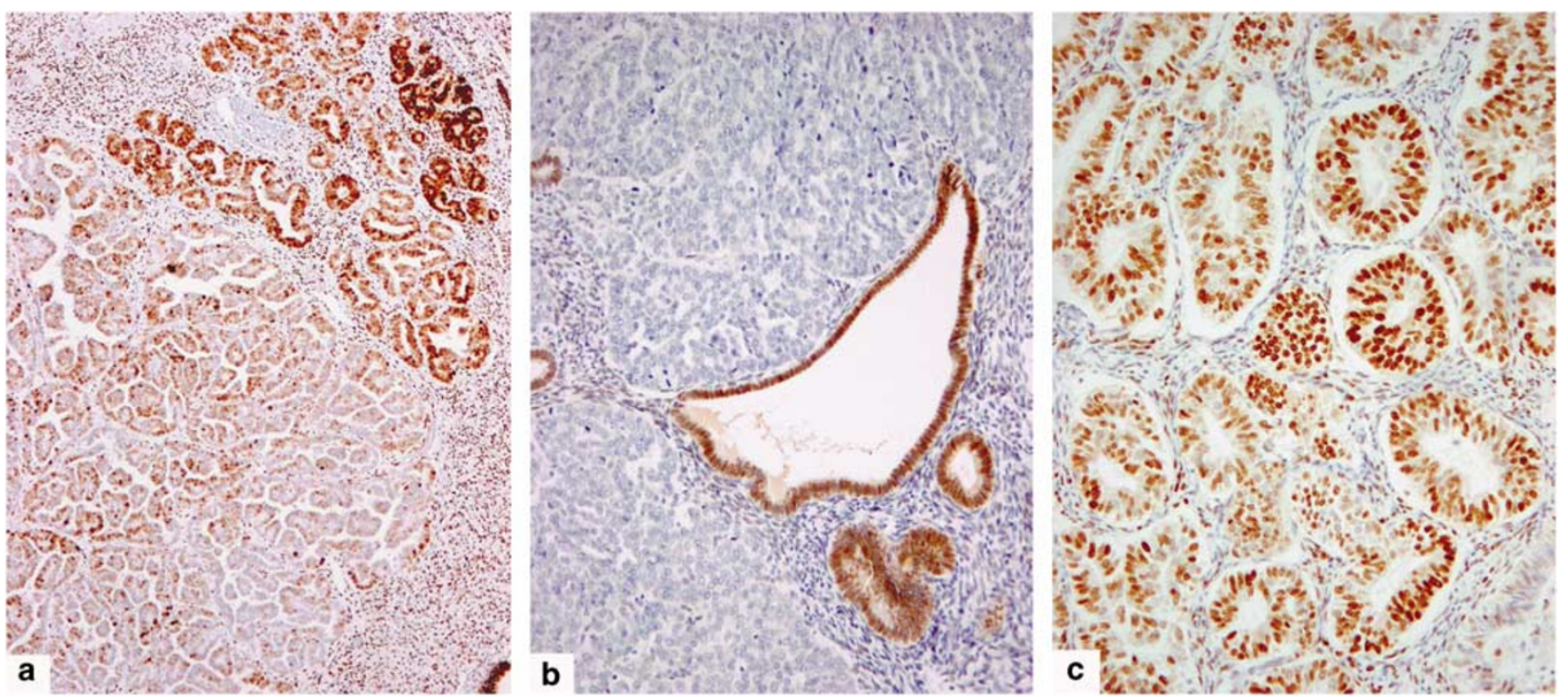

Figure 3 ER expression in endometrial cancer. (a) A serous carcinoma with focal ER expression. FIGO grade 3 endometrioid adenocarcinoma (b) is negative for ER (note contrasting ER-positive non-neoplastic elements), while (c) demonstrates a FIGO grade 1 endometrioid adenocarcinoma with diffuse ER expression.

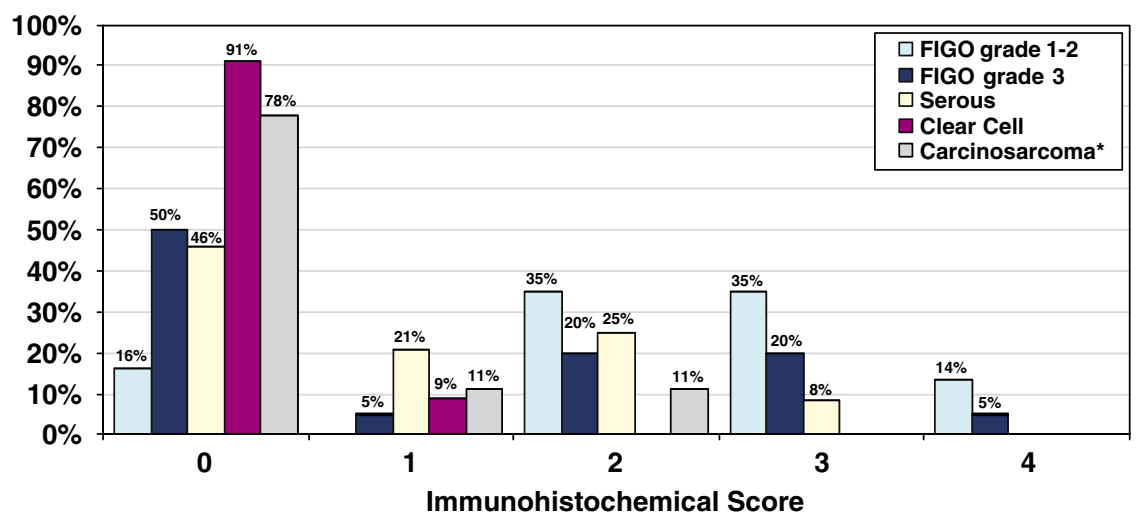

Figure 4 ER expression in endometrial cancer. FIGO, International Federation of Gynecology and Obstetrics. *Epithelial components only. 


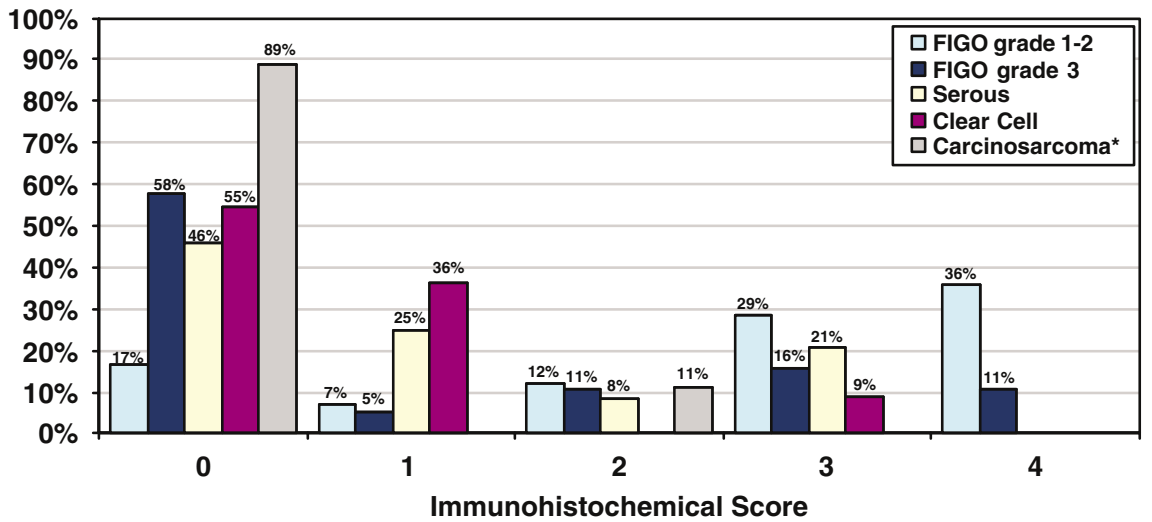

Figure 5 PR expression in endometrial cancer. FIGO, International Federation of Gynecology and Obstetrics. *Epithelial components only.
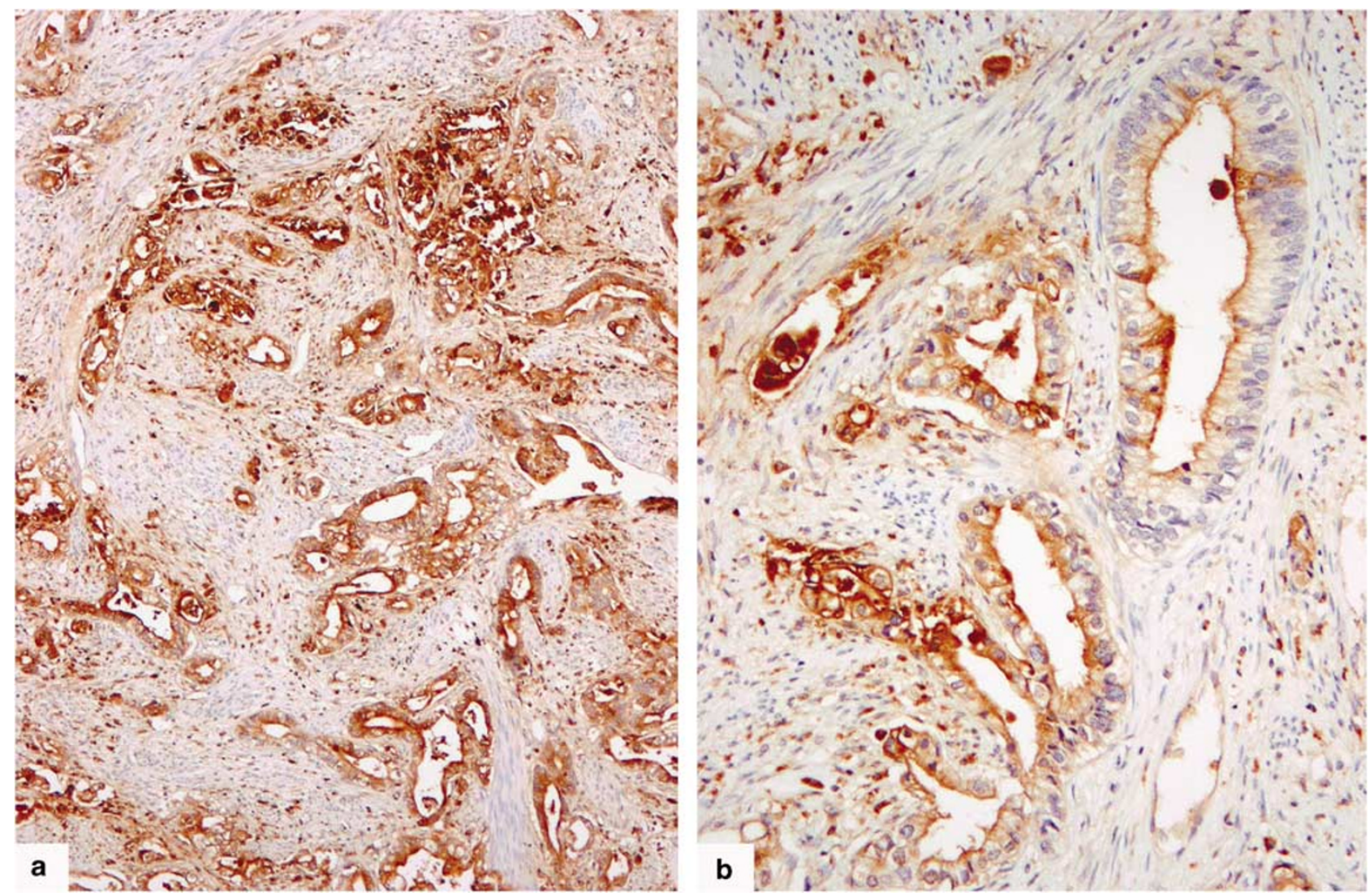

Figure 6 mCEA expression in endometrial cancer. Only rare endometrial adenocarcinomas showed diffuse cytoplasmic mCEA labeling (a, an endometrioid adenocarcinoma), which is reported to be relatively specific for endocervical adenocarcinoma. (b, another endometrioid adenocarcinoma) shows a more commonly encountered CEA labeling pattern in endometrial cancer, apical accentuation.

Monoclonal CEA (mCEA) (Figures 6 and 7): Most carcinomas were negative for mCEA. Small numbers of endometrioid adenocarcinomas, particularly those with mucinous differentiation, were focally CEA positive. The highest expression rates were seen in serous carcinomas and clear cell carcinomas (13 and $18 \%$, respectively); only rare cases were strongly immunoreactive. CEA immunoreactivity in endometrioid adenocarcinomas showed preferential staining of luminal cell membranes, but occasional cases also showed diffuse cytoplasmic coloration.

Vimentin (Figures 8 and 9): Vimentin was expressed in nearly every tumor, with rates ranging from $81 \%$ in FIGO grade 3 endometrioid carcinomas to $100 \%$ in carcinosarcomas. Despite the nearuniform rates of positivity for vimentin, there was a broad range of expression strength among tumors. Weakly positive tumors were uncommon, but scores 


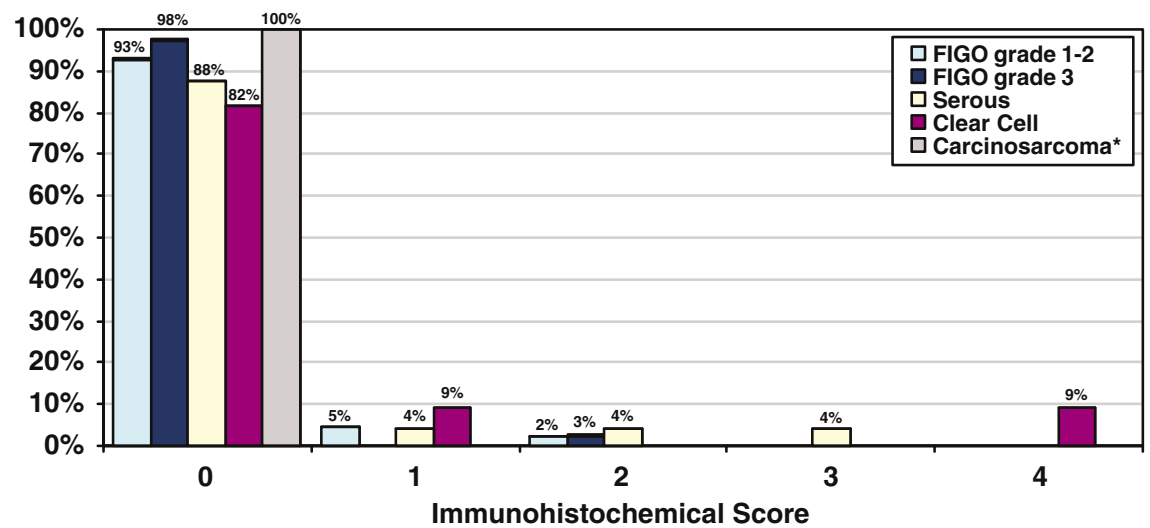

Figure 7 Monoclonal carcinoembryonic antigen (mCEA) expression in endometrial cancer. FIGO, International Federation of Gynecology and Obstetrics. *Epithelial components only.
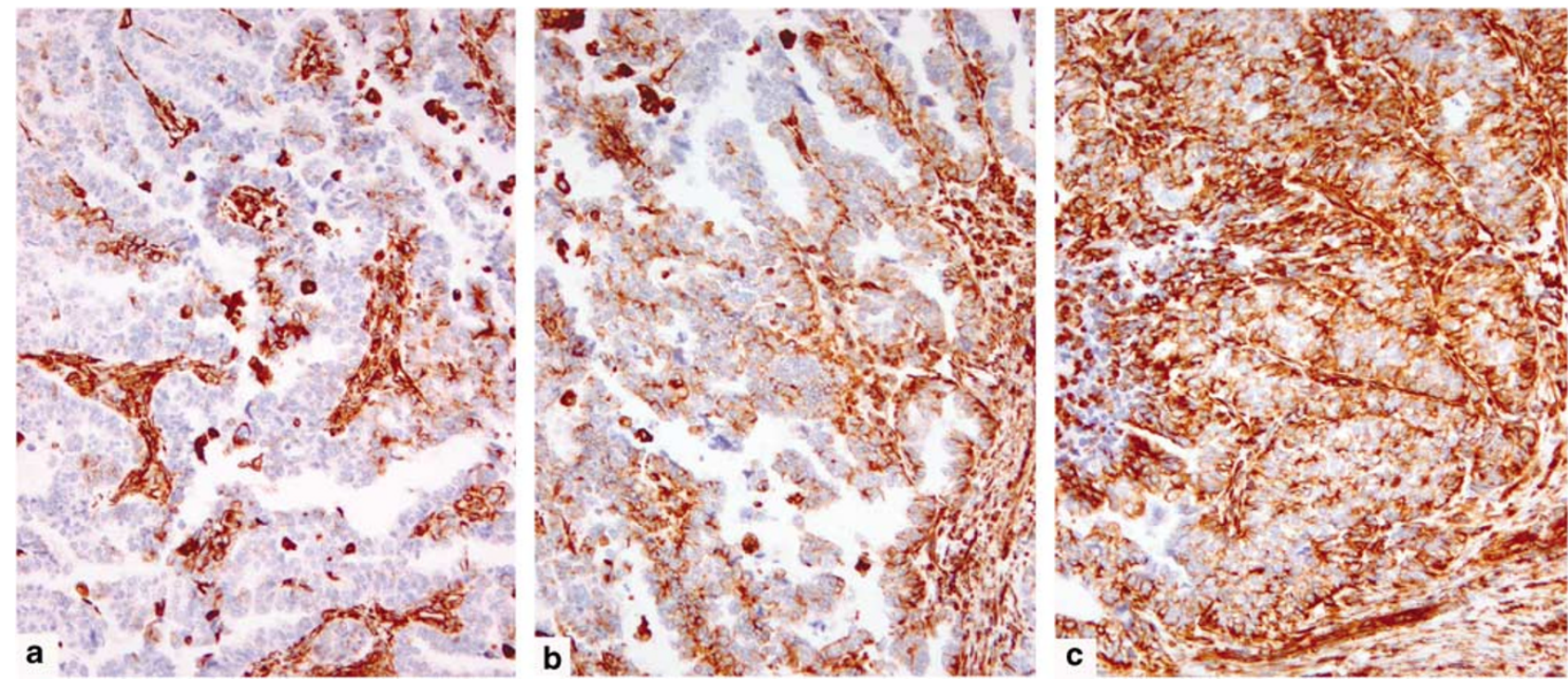

Figure 8 Vimentin expression in endometrial cancer. Rare endometrial cancers are negative for vimentin (a, a serous carcinoma) but the vast majority express vimentin either focally (b, serous carcinoma) or diffusely (c, an endometrioid adenocarcinoma).

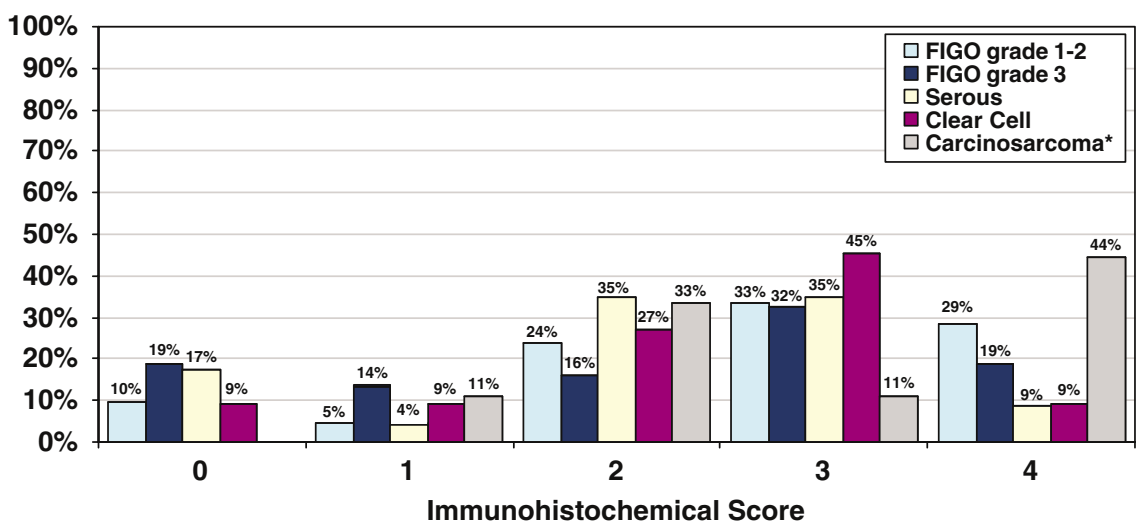

Figure 9 Vimentin expression in endometrial cancer. FIGO, International Federation of Gynecology and Obstetrics. ${ }^{*}$ Epithelial components only. 
Table 3 FIGO grades 1 and 2 endometrioid carcinoma immunophenotypes

\begin{tabular}{lccccc}
\hline p16 & ER & PR & mCEA & Vimentin & \% with this panel \\
\hline- & + & + & - & + & 70 \\
- & - & - & - & + & 8 \\
+ & + & + & - & + & 5 \\
- & - & + & - & + & 2.7 \\
- & - & + & + & - & 2.7 \\
- & + & - & - & + & 2.7 \\
- & + & + & - & - & 2.7 \\
- & + & + & + & + & 2.7 \\
+ & - & - & - & - & 2.7
\end{tabular}

FIGO, International Federation of Gynecology and Obstetrics; ER, estrogen receptor; PR, progesterone receptor; mCEA, monoclonal carcinoembryonic antigen.

Table 4 FIGO grade 3 endometrioid carcinoma immunophenotypes

\begin{tabular}{llllcc}
\hline p16 & ER & PR & mCEA & Vimentin & \% with this panel \\
\hline- & + & + & - & + & 26 \\
- & - & - & - & + & 20 \\
- & - & - & - & - & 11 \\
+ & - & - & - & + & 11 \\
- & - & + & - & + & 9 \\
+ & + & - & - & - & 9 \\
- & + & - & - & - & 2.9 \\
- & + & + & - & - & 2.9 \\
- & + & + & + & + & 2.9 \\
+ & + & + & - & + & \\
\hline
\end{tabular}

ER, estrogen receptor; PR, progesterone receptor; mCEA, monoclonal carcinoembryonic antigen.

Table 5 Serous carcinoma immunophenotypes

\begin{tabular}{lccccc}
\hline p16 & ER & PR & mCEA & vimentin & \% with this panel \\
\hline+ & - & + & - & + & 22 \\
+ & + & + & - & + & 17 \\
+ & - & - & - & + & 13 \\
+ & + & - & - & + & 13 \\
- & - & - & - & + & 9 \\
+ & + & - & - & - & 9 \\
+ & + & + & - & - & 4.4 \\
+ & - & - & + & + & 4.4 \\
+ & + & + & + & + &
\end{tabular}

ER, estrogen receptor; PR, progesterone receptor; mCEA, monoclonal carcinoembryonic antigen.

of 2-4 were seen in large numbers among every tumor type.

A summary of the most common immunophenotype for each endometrial carcinoma subtype is presented in Tables 3-7. The most common immunophenotype for FIGO grades 1 and 2 endometrioid carcinoma was p16 $(-)$, ER $(+)$, PR $(+)$, CEA $(-)$, and vimentin $(+)$. This phenotype was seen in $70 \%$ of FIGO grades 1 and 2 endometrioid carcinomas. This was also the most common immunophenotype encountered in FIGO grade 3 endometrioid carcinomas, but only $26 \%$ of such tumors demon-
Table 6 Clear cell carcinoma immunophenotypes

\begin{tabular}{lccccc}
\hline p16 & ER & PR & mCEA & Vimentin & \% with this panel \\
\hline+ & - & - & - & + & 27 \\
- & - & - & - & + & 18 \\
- & - & + & + & + & 18 \\
- & - & - & - & - & 9 \\
- & - & + & - & + & 9 \\
+ & - & + & - & + & 9 \\
+ & + & + & - & + & 9 \\
\hline
\end{tabular}

ER, estrogen receptor; PR, progesterone receptor; mCEA, monoclonal carcinoembryonic antigen.

Table 7 Carcinosarcoma ${ }^{\mathrm{a}}$ immunophenotypes

\begin{tabular}{llllcl}
\hline P16 & ER & PR & mCEA & vimentin & \% with this panel \\
\hline+ & - & - & - & + & 56 \\
- & - & - & - & + & 22 \\
- & + & + & - & + & 11 \\
+ & + & - & - & + & 11
\end{tabular}

ER, estrogen receptor; PR, progesterone receptor; mCEA, monoclonal carcinoembryonic antigen.

${ }^{\mathrm{a}}$ Epithelial components only.

strated this phenotype. A sizable proportion of tumors in the FIGO grade 3 category lacked ER and PR expression and many expressed p16. In all, $22 \%$ percent of serous carcinomas were p16 $(+)$, ER $(-)$, PR (+), CEA (-), and vimentin $(+)$-the most common immunophenotype among serous carcinomas. Occasional serous carcinomas also showed ER expression. The most common clear cell carcinoma immunophenotype, seen in $27 \%$ of such tumors, was p16 (+), ER (-), PR (-), CEA (-), and vimentin $(+)$. Many clear cell carcinomas, however, lacked p16 expression. Clear cell carcinoma was the only endometrial cancer tumor type in which CEA expression appeared in a top-three immunophenotype. The majority of carcinosarcomas $(56 \%)$ were p16 $(+)$, ER $(-)$, PR $(-)$, CEA $(-)$, and vimentin $(+)$.

\section{Discussion}

Our results demonstrate that the immunophenotypes of endometrial cancers are diverse, indicating that using immunohistochemistry to define the site of origin of a tumor in a curettage specimen can be complicated and subject to error. We also encountered wide variations in distribution and intensity of expression with each marker. p16 expression was common in the epithelial component of endometrial carcinosarcomas and endometrial clear cell carcinomas and was ubiquitous in serous carcinomas where the expression was typically strong and diffuse. Although ER and PR were expressed in many endometrioid adenocarcinomas, a significant proportion of these tumors were negative. Our data also confirmed that CEA is only rarely expressed in any endometrial carcinoma and that vimentin 
expression is typical of endometrial carcinomas regardless of histologic subtype; however, there were large numbers of endometrial cancers that expressed vimentin only focally or in a patchy distribution.

p16 is a protein encoded by p16INK4a. It normally blocks progression of the cell cycle by inhibiting CDK complex formation with the retinoblastoma protein (RB). ${ }^{12-14}$ It may be inactivated by mutation or by promoter hypermethylation. In gynecologic specimens, p16 immunohistochemistry has been used as an indirect assay for HPV infection ${ }^{1,15,16}$ and an even more indirect method of determining the primary site of origin ${ }^{1-3}$ (in human papillomavirus (HPV)-associated cervical cancers, viral oncoproteins E6 and E7 bind activated RB with consequent upregulation of p16 and promotion of DNA synthesis ${ }^{15,17}$ ). Well-recognized problems with this approach have been published; p16 expression has been described in non-neoplastic ciliated cells, the cells of tuboendometrioid metaplasia ${ }^{16,18,19}$ and even in endometrial cancer. ${ }^{1,2,20}$ In endometrial cancer, the expression pattern is generally described as weak and patchy, in contrast to the strong immunoreaction typically encountered in endocervical adenocarcinomas of the usual type. ${ }^{1,2}$ The mechanism of p16 expression in metaplastic cells and endometrial cancer has not been determined. We confirmed the generally weak and focal expression pattern in FIGO grades 1 and 2 endometrioid carcinomas, but also noted stronger expression in FIGO grade 3 endometrioid carcinomas, clear cell carcinomas and in the epithelial component of carcinosarcomas. Serous carcinomas showed strong and diffuse expression of $\mathrm{p} 16$, suggesting this could be exploited for the differential diagnosis with endometrioid adenocarcinoma. This should be studied in greater detail.

ER and PR expression are known to be common in well-differentiated endometrioid adenocarcinomas of endometrium, although less has been published about ER and PR expression in FIGO grade 3 endometrioid adenocarcinomas, ${ }^{21}$ clear cell carcinomas, ${ }^{22-24}$ and carcinosarcomas. ${ }^{25,26}$ Reports of ER and PR expression in serous carcinoma are not uniform. Serous carcinoma was erroneously considered a largely ER- and PR-positive tumor in the preimmunohistochemistry era. This was due to methods that failed to discriminate between ER/ PR-positive stromal elements and carcinoma. ${ }^{27-29}$ Following that, serous carcinoma began to be described as a tumor largely negative for ER/PR; ${ }^{30-32}$ this highlighted its distinction from most endometrioid carcinomas and conformed to the dualistic endometrial carcinogenesis model of Bokhman. ${ }^{33}$ Our data here suggest that ER/PR values for serous carcinoma are intermediate. Admittedly, while overall rates of ER/PR expression in serous carcinoma are generally comparable to that of FIGO grade 3 endometrioid carcinomas, which has been reported previously, ${ }^{21}$ the intensity and staining distribution of positive cases was quantitatively and qualitatively less. The epithelial component of carcinosarcoma was only very rarely positive for ER/PR, and ER expression was very uncommon in clear cell carcinoma. If an inappropriate emphasis is placed on immunohistochemistry results, the sufficient numbers of ER/PR-negative cases in each category might cause diagnostic difficulties with histologically similar adenocarcinomas, notably endocervical adenocarcinoma.

CEA has been touted as a good discriminatory marker for endometrial carcinoma vs histologic mimics, including endocervical carcinoma, ${ }^{4-7,34}$ because endometrial carcinoma is usually CEA-negative and endocervical carcinoma is usually positive. Dallenbach-Hellweg et al, ${ }^{5}$ however, reported that both endometrial and endocervical mucinous carcinomas expressed CEA; this was refuted by Kamoi et $a l,{ }^{9}$ who reported significantly more CEA expression in endocervical adenocarcinomas of the usual type compared to endometrioid adenocarcinomas of endometrium and endocervix and mucinous endometrial adenocarcinomas. The type of antibody used (polyclonal vs monoclonal) has some significance. According to Dabbs et $a{ }^{6}{ }^{6}$ although endometrial adenocarcinomas were largely negative for CEA, the rate of CEA positivity varied in endocervical adenocarcinomas, with significantly higher rates observed with monoclonal CEA as compared to polyclonal CEA. In our study, the only endometrial carcinoma histologic subtypes that expressed CEA in any significant numbers were clear cell and serous carcinomas. Expression in clear cell carcinomas has also been reported by Dallenbach-Hellweg et al. ${ }^{5}$ With only rare exceptions, positive cases here contained only scattered and weakly immunoreactive cells, most in a membrane distribution. McCluggage et $\mathrm{al}^{7}$ reported membrane expression of mCEA in the glandular component of endometrioid carcinomas of the endometrium that contrasted with the much more common cytoplasmic localization in endocervical adenocarcinomas. Unlike ER, PR, and p16, which showed significant variability in expression between subtypes of endometrial cancers, CEA expression was more uniformly negative, suggesting that it is a better candidate for a site-specific marker.

Vimentin is characteristically positive in endometrial cancer, another point that our study confirms. Of interest is the very heterogeneous distribution and staining patterns seen in many cancers; a number of cases showed only focal and weak vimentin staining. This point calls into question its value as a discriminatory marker for gynecologic carcinomas represented in biopsy or curettage material.

Endometrial cancers demonstrate substantial immunophenotypic diversity that remained apparent even within groups of similar histologic subtype and grade. ER, PR, and p16 expression was more illustrative of tumor type and degree of differentiation than they were of endometrial origin. 
In contrast, the vimentin-positive/CEA-negative phenotype remained the most constant among all endometrial cancers. These data underscore some of the problems that result from over-reliance on immunohistochemistry when differentiating between endometrial and endocervical adenocarcinomas, particularly. Traditional methods, including detailed morphologic study and clinical and radiologic correlation, should not be discounted.

\section{References}

1 Ansari-Lari MA, Staebler A, Zaino RJ, et al. Distinction of endocervical and endometrial adenocarcinomas: immunohistochemical p16 expression correlated with human papillomavirus (HPV) DNA detection. Am J Surg Pathol 2004;28:160-167.

2 McCluggage WG, Jenkins D. p16 immunoreactivity may assist in the distinction between endometrial and endocervical adenocarcinoma. Int J Gynecol Pathol 2003;22:231-235.

3 Negri G, Egarter-Vigl E, Kasal A, et al. p16INK4a is a useful marker for the diagnosis of adenocarcinoma of the cervix uteri and its precursors: an immunohistochemical study with immunocytochemical correlations. Am J Surg Pathol 2003;27:187-193.

4 Wahlstrom T, Lindgren J, Korhonen M, et al. Distinction between endocervical and endometrial adenocarcinoma with immunoperoxidase staining of carcinoembryonic antigen in routine histological tissue specimens. Lancet 1979;2:1159-1160.

5 Dallenbach-Hellweg G, Lang-Averous G, Hahn U. The value of immunohistochemistry in the differential diagnosis of endometrial carcinomas. APMIS Suppl 1991;23:91-99.

6 Dabbs DJ, Sturtz K, Zaino RJ. The immunohistochemical discrimination of endometrioid adenocarcinomas. Hum Pathol 1996;27:172-177.

7 McCluggage WG, Sumathi VP, McBride HA, et al. A panel of immunohistochemical stains, including carcinoembryonic antigen, vimentin, and estrogen receptor, aids the distinction between primary endometrial and endocervical adenocarcinomas. Int J Gynecol Pathol 2002;21:11-15.

8 Staebler A, Sherman ME, Zaino RJ, et al. Hormone receptor immunohistochemistry and human papillomavirus in situ hybridization are useful for distinguishing endocervical and endometrial adenocarcinomas. Am J Surg Pathol 2002;26:998-1006.

9 Kamoi S, AlJuboury MI, Akin MR, et al. Immunohistochemical staining in the distinction between primary endometrial and endocervical adenocarcinomas: another viewpoint. Int J Gynecol Pathol 2002;21:217-223.

10 Kononen J, Bubendorf L, Kallioniemi A, et al. Tissue microarrays for high-throughput molecular profiling of tumor specimens. Nat Med 1998;4:844-847.

11 Hedvat CV, Jaffe ES, Qin J, et al. Macrophage-derived chemokine expression in classical Hodgkin's lymphoma: application of tissue microarrays. Mod Pathol 2001;14:1270-1276.

12 Sherr CJ. The INK4a/ARF network in tumour suppression. Nat Rev Mol Cell Biol 2001;2:731-737 (Review).

13 Sherr CJ, McCormick F. The RB and p53 pathways in cancer. Cancer Cell 2002;2:103-112 (Review).
14 Lowe SW, Sherr CJ. Tumor suppression by Ink4a-Arf: progress and puzzles. Curr Opin Genet Dev 2003;13:77-83 (Review).

15 Sano T, Oyama T, Kashiwabara K, et al. Expression status of p16 protein is associated with human papillomavirus oncogenic potential in cervical and genital lesions. Am J Pathol 1998;153:1741-1748.

16 Riethdorf L, Riethdorf S, Lee KR, et al. Human papillomaviruses, expression of p16, and early endocervical glandular neoplasia. Hum Pathol 2002;33:899-904.

17 Sano T, Oyama T, Kashiwabara K, et al. Immunohistochemical overexpression of p16 protein associated with intact retinoblastoma protein expression in cervical cancer and cervical intraepithelial neoplasia. Pathol Int 1998;48:580-585.

18 Cameron RI, Maxwell P, Jenkins D, et al. Immunohistochemical staining with MIB1, bcl2 and p16 assists in the distinction of cervical glandular intraepithelial neoplasia from tubo-endometrial metaplasia, endometriosis and microglandular hyperplasia. Histopathology 2002;41:313-321.

19 Murphy N, Heffron CC, King B, et al. p16INK4A positivity in benign, premalignant and malignant cervical glandular lesions: a potential diagnostic problem. Virchows Arch 2004;445:610-615.

20 Semczuk A, Boltze C, Marzec B, et al. p16INK4A alterations are accompanied by aberrant protein immunostaining in endometrial carcinomas. J Cancer Res Clin Oncol 2003;129:589-596.

21 Soslow RA, Shen PU, Chung MH, et al. Cyclin D1 expression in high-grade endometrial carcinomasassociation with histologic subtype. Int J Gynecol Pathol 2000;19:329-334.

22 Lax SF, Pizer ES, Ronnett BM, et al. Clear cell carcinoma of the endometrium is characterized by a distinctive profile of p53, Ki-67, estrogen, and progesterone receptor expression. Hum Pathol 1998;29: $551-558$.

23 Vang R, Whitaker BP, Farhood AI, et al. Immunohistochemical analysis of clear cell carcinoma of the gynecologic tract. Int J Gynecol Pathol 2001;20: 252-259.

24 Cameron RI, Ashe P, O'Rourke DM, et al. A panel of immunohistochemical stains assists in the distinction between ovarian and renal clear cell carcinoma. Int J Gynecol Pathol 2003;22:272-276.

25 Lantta M, Karkkainen J, Wahlstrom T, et al. Estradiol and progesterone receptors in gynecologic sarcomas. Acta Obstet Gynecol Scand 1984;63:505-508.

26 Ansink AC, Cross PA, Scorer P, et al. The hormonal receptor status of uterine arcinosarcomas (mixed mullerian tumours): an immunohistochemical study. J Clin Pathol 1997;50:328-331.

27 Carcangiu ML, Chambers JT, Voynick IM, et al. Immunohistochemical evaluation of estrogen and progesterone receptor content in 183 patients with endometrial carcinoma. Part I: Clinical and histologic correlations. Am J Clin Pathol 1990;94:247-254.

28 Chambers JT, Carcangiu ML, Voynick IM, et al. Immunohistochemical evaluation of estrogen and progesterone receptor content in 183 patients with endometrial carcinoma. Part II: Correlation between biochemical and immunohistochemical methods and survival. Am J Clin Pathol 1990;94:255-260.

29 Umpierre SA, Burke TW, Tornos C, et al. Immunocytochemical analysis of uterine papillary serous 
carcinomas for estrogen and progesterone receptors. Int J Gynecol Pathol 1994;13:127-130.

30 Koshiyama M, Konishi I, Wang DP, et al. Immunohistochemical analysis of p53 protein over-expression in endometrial carcinomas: inverse correlation with sex steroid receptor status. Virchows Arch A Pathol Anat Histopathol 1993;423:265-271.

31 Demopoulos RI, Mesia AF, Mittal K, et al. Immunohistochemical comparison of uterine papillary serous and papillary endometrioid carcinoma: clues to pathogenesis. Int J Gynecol Pathol 1999;18:233-237.
32 Darvishian F, Hummer AJ, Thaler HT, et al. Serous endometrial cancers that mimic endometrioid adenocarcinomas: a clinicopathologic and immunohistochemical study of a group of problematic cases. Am J Surg Pathol 2004;28:1568-1578.

33 Bokhman JV. Two pathogenetic types of endometrial carcinoma. Gynecol Oncol 1983;15:10-17.

34 Castrillon DH, Lee KR, Nucci MR. Distinction between endometrial and endocervical adenocarcinoma: an immunohistochemical study. Int J Gynecol Pathol 2002;21:4-10. 